\title{
STOCHASTIC LOCAL LOAD REDISTRIBUTION IN THE FIBRE BUNDLE MODEL OF NANOPILLAR ARRAYS
}

\author{
Tomasz Derda \\ Institute of Mathematics, Czestochowa University of Technology \\ Częstochowa, Poland \\ tomasz.derda@im.pcz.pl
}

\begin{abstract}
We study the breakdown of the nanopillar arrays subjected to axial loading. The pillar-strength-thresholds are drawn from a given probability distribution. Pillars are located in the nodes of the supporting regular lattice. In this work we introduce stochastic local load sharing - after pillar breakdown each of its nearest intact neighbours obtains a random fraction of the failing load. Two types of loading procedure are employed, namely quasi-static and finite force. We analyse critical loads, catastrophic avalanches as well as probabilities of cascade and breakdown.
\end{abstract}

Keywords: nanopillars, fibre bundle model, stochastic load redistribution, skew normal distribution, local load sharing

\section{Introduction}

The modelling of damage processes is an important issue in the community of physicists and engineers. Particularly fracture and damage of heterogeneous materials are the problems which have attracted an intensive research over the last decades [1-3]. Among the proposed statistical models for failure of heterogeneous materials, one is fundamental, namely the fibre bundle model (FBM) [3-6]. The approach based on the FBM, yet very simple, is able to capture the most important properties of material damage and breakdown in disordered media - various loading conditions, types of load sharing and damage mechanisms can be used. In the FBM a material is visible as a discrete set of parallel fibres with stochastic strength thresholds. Under a load exceeding its strength threshold a fibre breaks and the stress carried by this fibre is redistributed to other fibres according to a given load transfer rule. In a global load sharing rule the stress is equally transferred to all remaining intact fibres. On the other hand, in the case of the local load sharing rule, the stress is equally redistributed to the nearest intact neighbours of the broken one. Other rules and mixtures of them are also proposed [7].

Inspired by experimental compression tests of metallic micro- and nano-sized pillars [8-10], we applied the fibre bundle approach to simulate the breakdown 
in arrays of nanopillars assembled perpendicularly to a flat substrate [11-13]. In this paper we propose a stochastic version of the local load sharing rule. As far as we know, two other models of stochastic load redistribution are investigated in the literature. In the first one it is assumed that the load from the broken element is redistributed to a fixed number of randomly chosen intact elements [14]. In the second model each intact element obtains a random fraction of the load of the damaged element [15]. In our model we restrict the randomness of load redistribution to the nearest neighbourhood only.

\section{Model of nanopillar array with stochastic load sharing}

We shall consider an array of $N$ longitudinal nanopillars arranged on a twodimensional lattice. In this paper only regular arrangements are analysed, namely hexagonal, square and triangular symmetries. Figure 1 illustrates regular lattices represented by nodes and edges connecting neighbouring nodes. The distance between two nodes is calculated as the number of edges in the shortest possible path between these nodes.
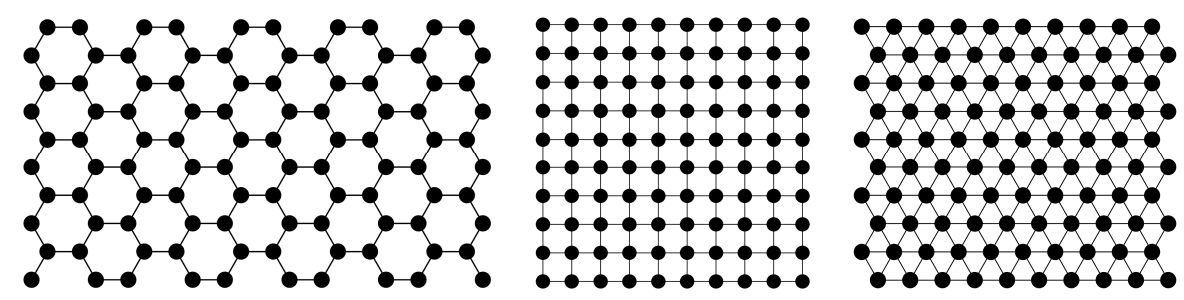

Fig. 1. Regular lattice geometries: hexagonal (left), square (centre), triangular (right). Each exemplary configuration consists of 100 nodes

Due to various defects during fabrication the nanopillars are characterised by strength thresholds (also called critical loads) $\sigma_{t h}^{i}, i=1,2, . ., N$, which are quenched random variables. It is assumed that the randomness of the strength thresholds represents the disorder of heterogeneous materials. Strength thresholds are typically distributed according to uniform or Weibull distributions. Uniform distribution has the probability density and cumulative distribution functions: $p\left(\sigma_{t h}\right)=1$ and $P\left(\sigma_{t h}\right)=\sigma_{t h}$, respectively. According to the uniform distribution the pillar-strength-thresholds are drawn from the interval $[0,1]$. The second employed distribution is the Weibull distribution with density:

$$
p\left(\sigma_{t h}\right)=\frac{\rho}{\lambda}\left(\frac{\sigma_{t h}}{\lambda}\right)^{\rho-1} \exp \left\{-\left(\frac{\sigma_{t h}}{\lambda}\right)^{\rho}\right\}
$$


and distribution function:

$$
P\left(\sigma_{t h}\right)=1-\exp \left\{-\left(\frac{\sigma_{t h}}{\lambda}\right)^{\rho}\right\}
$$

where $\rho$ and $\lambda$ are the Weibull index and scale parameter, respectively. It is assumed that $\lambda=1$. Weibull index controls the degree of disorder in the system. As the Weibull index is increased, the disorder degree decreases. Weibull index $\rho$ typically varies between 2 and 10 .

In this paper we also employ uniform and Weibull distributions truncated below a given initial load per pillar $\sigma_{0}[15]$ :

$$
\begin{gathered}
p\left(\sigma_{t h}\right)=\left\{\begin{array}{cc}
\frac{1}{1-\sigma_{0}}, & \text { if } \sigma_{0} \leq \sigma_{t h} \leq 1, \\
0 & \text { else. }
\end{array}\right. \\
p\left(\sigma_{t h}\right)=\left\{\begin{array}{cc}
\rho \sigma_{t h}^{\rho-1} \exp \left(\sigma_{0}^{\rho}-\sigma_{t h}^{\rho}\right), & \text { if } \sigma_{0} \leq \sigma_{t h}, \\
0 & \text { else. }
\end{array}\right.
\end{gathered}
$$

The nanopillar array is subjected to an external axial load $F$. Before the application of load, all nanopillars in the system are intact. We employ two different, but equivalent procedures of loading. The first one is a quasi-static loading. In this approach the initial load equals zero $(F=0)$. Then the external load is uniformly increased on all intact pillars until the weakest pillar breaks. The destruction of a pillar, caused by a load attaining its strength threshold, is instantaneous and irreversible. After the breakdown of the weakest pillar, the increase of external load stops and load from the damaged pillar is transferred to other surviving pillars in the system according to a given load transfer rule. We apply the stochastic local load sharing, i.e. the load coming from the damaged pillar is redistributed in random fractions to its nearest surviving neighbours. If the damaged pillar has $M$ intact neighbours, the load $\sigma_{k}^{t r}$ transferred to the $k$-th neighbour can be described by the formula:

$$
\sigma_{k}^{t r}=\sigma_{d} \frac{\delta_{k}}{\sum_{j=1}^{M} \delta_{j}}
$$

Here, factors $\delta_{j}, j=1,2, \ldots, M$ are random numbers drawn according to a uniform distribution on the interval $[0,1], \sigma_{d}$ is the load carried by the damaged pillar. 
Load redistribution increases stresses on the intact neighbours of a just damaged pillar - it may provoke further failures and, after the next load transfers, possible cascades of subsequent failures. If the load transfer does not cause further failures, it means that equilibrium state is reached. In such a stable state the external load is again uniformly increased on all intact pillars until the destruction of the weakest pillar. The above described procedure is repeated up to the failure of all pillars in the set.

Loading of the system can also be realised using an external force which is kept constant during the entire loading process. All pillars with strength thresholds smaller than $F / N$ are destroyed immediately after application of force $F$. The load from destroyed pillars is redistributed according to a stochastic local load sharing.

\section{Results of the numerical simulations}

\subsection{Critical loads and catastrophic avalanches in quasi-static loading}

For a quasi-static loading procedure, the destruction of the system proceeds in an avalanche-like manner. Load increase induces failure of one or more pillars and the next load increase provokes subsequent failures etc. Hence, the number of damaged pillars between two consecutive load increments is called an avalanche $(\Delta)$. It follows that each avalanche is initiated by increase of external load.

Many cascades of simultaneous pillar crashes appear during the final stage of the system damage. These cascades, leading to a complete system breakdown, form the catastrophic (critical) avalanche $\Delta_{c}$. The emergence of the catastrophic avalanche is triggered by attaining critical load $F_{c}$. This means that the first cascade in the critical avalanche is initiated by the external load increase, the next cascades are induced only by internal stress redistribution. In the following we examine critical loads and catastrophic avalanches.

In numerical simulations the cycle of a complete system breakdown was performed many times in order to obtain reliable results. For each simulation pillar-strength-thresholds were drawn separately.

In order to compare results for different system sizes, the catastrophic avalanche sizes and critical loads have been scaled by the appropriate initial system sizes: $\Delta_{c} / N$ and $\sigma_{c}=F_{c} / N$.

Figure 2 illustrates results of the mean critical loads $\left\langle\sigma_{c}\right\rangle$ for three different lattice geometries. For all analysed arrangements $\left\langle\sigma_{c}\right\rangle$ is a monotonically decreasing function of the system size $N$ - this is in accordance with our previous results for the classical LLS rule [12,13]. Taking into consideration lattice geometry, there is an ordering: hexagonal (weakest), square, triangular (strongest). Arrangement of 
pillars in the nodes of a given lattice is associated with a specific number of nearest neighbours. In hexagonal arrangement the pillar has three nearest neighbours, square arrangement - four, triangular arrangement - six. The bigger the number of nearest neighbours, the more dispersed transfer from destroyed pillar. For this reason the triangular arrangement is the strongest one. From Figure 2 it is also seen that a square system with a stochastic local load transfer is significantly weaker than a square system with classical local load sharing. It can be explained as follows: in the classical model load from destroyed pillar is equally redistributed to its nearest intact neighbours. For instance, if the damaged pillar has four nearest intact neighbours, each of these neighbours obtains one quarter of its load. For our stochastic model, in such a situation, each of the neighbours obtains on average one quarter of its load. However, some neighbours obtain more than one quarter, others less than one quarter. For example, there is almost $30 \%$ probability that the intact neighbour obtains at least one third of the load from the destroyed pillar. It increases the failure probability of these neighbouring pillars and, in consequence, the probability of subsequent pillar failures rises.

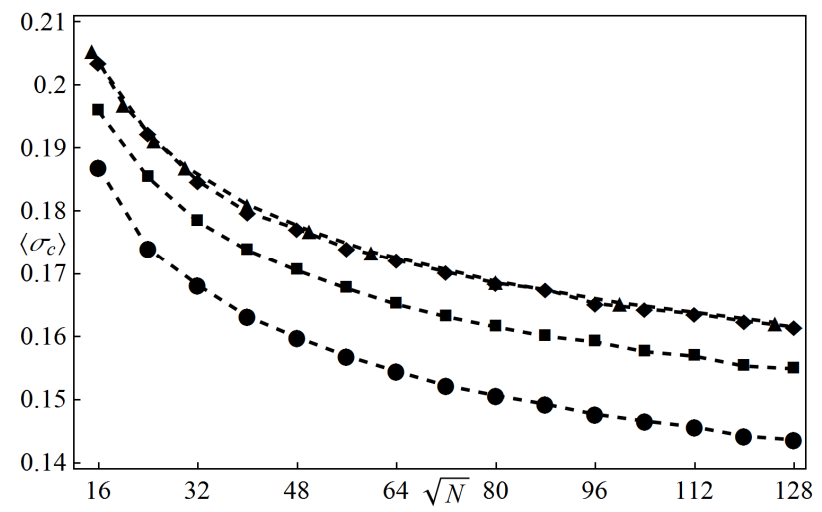

Fig. 2. The mean critical load $\left\langle\sigma_{c}\right\rangle$ versus square root of the system size $\sqrt{N}$ for different system arrangements: hexagonal (circles), square (squares), triangular (diamonds). Triangles represent results for the classical LLS rule on a square lattice. The averages are taken from at least 1000 samples for each presented value. Uniform distribution of strength thresholds is applied

Figure 3 presents the mean sizes of the normalized catastrophic avalanches. All regular pillar arrangements can be compared: the ordering reported for the critical loads is reversed. Normalized catastrophic avalanche sizes rise with increasing system size. This is consistent with results obtained for the classical LLS rule $[12,13]$. It is seen from Figures 2 and 3 that results for the classical LLS rule on square lattice and for the stochastic LLS rule on triangular lattice are almost the same. 


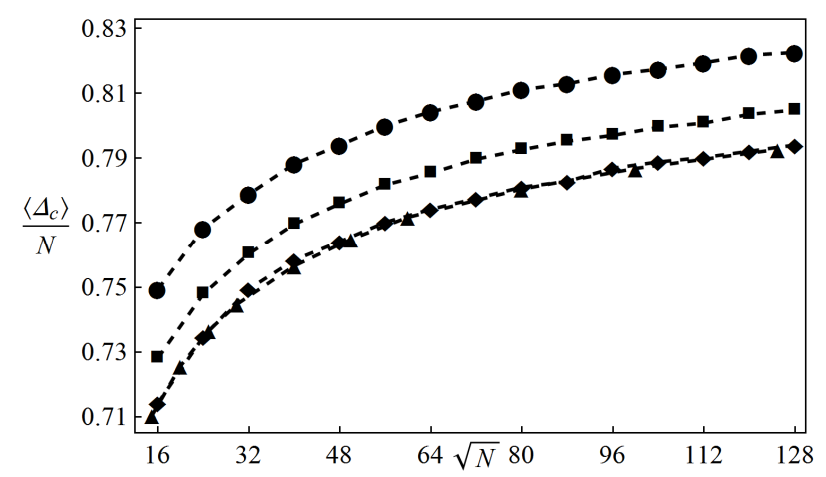

Fig. 3. The mean size of the critical avalanche $\left\langle\Delta_{c}\right\rangle$ scaled by the number of pillars versus the square root of the system size $\sqrt{N}$ for different system arrangements: hexagonal (circles), square (squares), triangular (diamonds). Triangles represent results for the classical LLS rule on a square lattice. The averages are taken from at least 1000 samples for each presented value. Uniform distribution of strength thresholds is applied

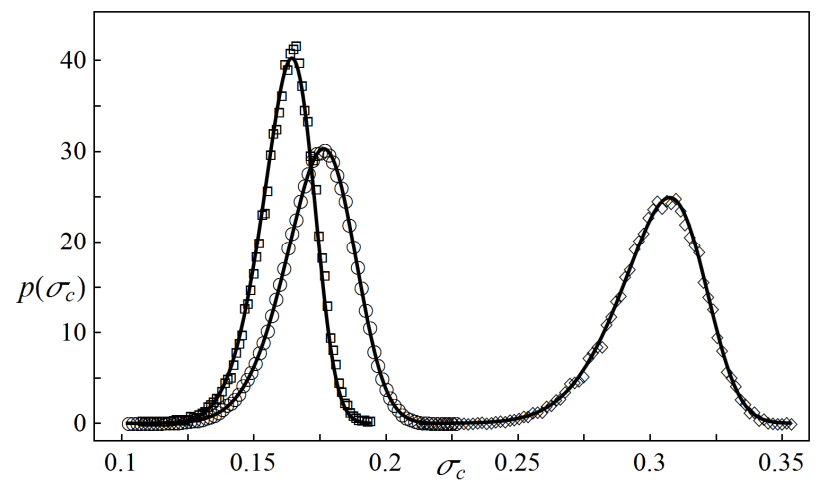

Fig. 4. Empirical probability density functions of the $\sigma_{c}$ in an array with: $40 \times 40$ (uniform distribution, circles), $80 \times 80$ (uniform distribution, squares) and $80 \times 80$ pillars (Weibull distribution with index $\rho=2$, diamonds). The solid lines represent skew-normally distributed $\sigma_{c}$ with parameters computed from the samples

In order to investigate distributions of $\sigma_{c}$ and $\Delta_{c} / N$ we have performed a series of simulations for the following square arrangements: $40 \times 40$ (uniform distribution, 475000 samples), $80 \times 80$ (uniform and Weibull distributions, 30000 samples). Empirical probability density distributions of $\sigma_{c}$ and $\Delta_{c} / N$ are graphically reported in Figures 4 and 5, respectively. Distributions of $\sigma_{c}$ and $\Delta_{c} / N$ follow three-parameter skew normal distribution, which is a generalisation of normal distribution allowing for non-zero skewness. The distribution of $\sigma_{c}$ has a negative skewness, while $\Delta_{c} / N$ is characterised by a positively skewed distribution. 


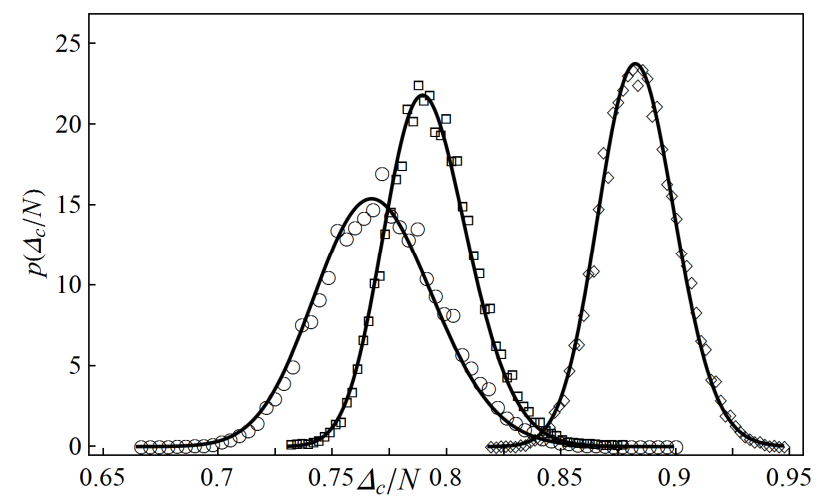

Fig. 5. Empirical probability density functions of the $\Delta_{c} / N$ in an array with: $40 \times 40$ (uniform distribution, circles), $80 \times 80$ (uniform distribution, squares) and $80 \times 80$ pillars (Weibull distribution with index $\rho=2$, diamonds). The solid lines represent skew-normally distributed $\Delta_{c} / N$ with parameters computed from the samples

\subsection{Probabilities of breakdown and cascade for fixed external load}

In this subsection we analyse the results of damage processes in nanopillar arrays loaded by finite force $F$. Application of finite force on the nanopillar array can lead to a partial damage - the system reaches an equilibrium after cascades of the pillar crashes. Cascades may also be self-sustained until complete system failure. Besides, there is a probability of no-cascade - all pillars in the array have strength thresholds bigger than $\sigma=F / N$.

Two classes of pillar-strength-threshold distributions are considered, namely distributions without and with truncation. We assume that the initial load $\sigma=F / N$ of all pillars is equal. Systems with non-truncated distributions are subjected to various external forces $F$, whereas the initial pillar stress in arrays with distributions truncated below $\sigma_{0}$ is always equal to $\sigma_{0}=F / N$. Furthermore, in the latter, the weakest pillar has strength-threshold $\sigma_{t h}=\sigma_{0}$. It ensures destruction of at least one pillar.

We focus on the calculation of the following quantities:

- cascade probability $P_{c}$ - the probability that an initial pillar destruction induces subsequent pillar failures. It is reasonable to compute this quantity for systems with truncated distributions,

- breakdown probability $P_{b}$ - application of force $F$ leads to the destruction of all pillars i.e. complete breakdown of the system.

Figures 6 and 7 present empirical breakdown probabilities of systems with pillar-strength-thresholds drawn according to non-truncated distributions. We observe dependence between breakdown probability and the number of nearest neighbours in the given lattice: the bigger the number of nearest neighbours, the smaller 
the probability of system breakdown under a given load (see Fig. 6). However, this dependence is restricted to some interval between loads too small to provoke complete failure of any arrangement and loads large enough to damage all arrangements. From Figure 7 it is seen that breakdown probability under given initial load $\sigma$ increases as the system size is increased. It can be concluded that these two dependencies are consistent with the results of critical loads discussed in the previous subsection.

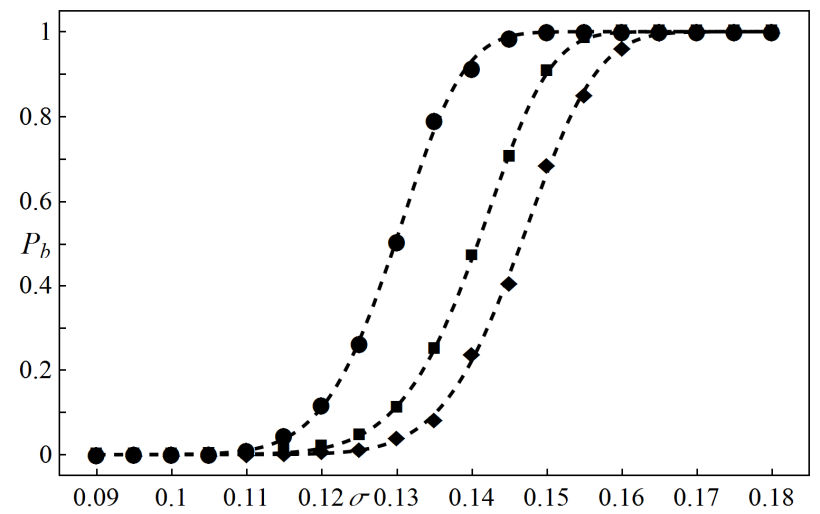

Fig. 6. Empirical breakdown probability $P_{b}$ as a function of initial load per pillar $\sigma$ for different pillar arrangements: hexagonal (circles), square (squares) and triangular (diamonds). Arrays consist of $128^{2}$ pillars with uniformly distributed strength thresholds. 1000 samples were taken for each presented value. The dashed lines represent function (6) with parameters computed from simulations

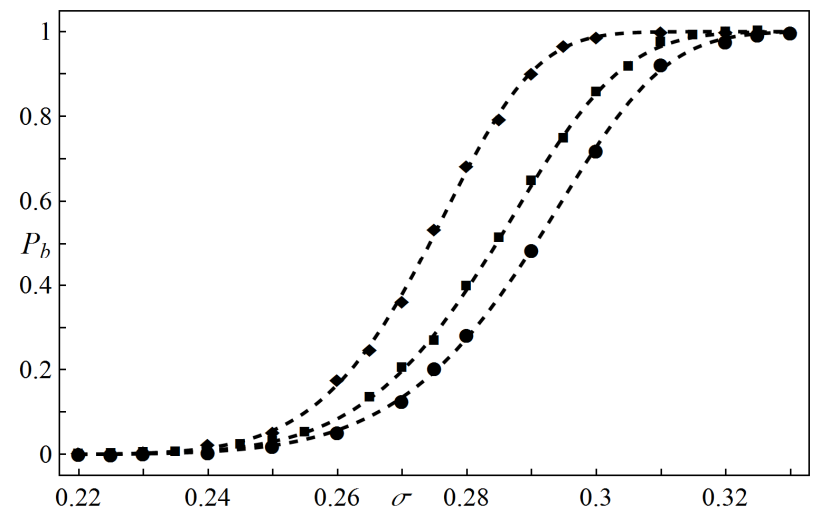

Fig. 7. Empirical breakdown probability $P_{b}$ as a function of initial load per pillar $\sigma$ for different system sizes: $64 \times 64$ (circles), $80 \times 80$ (squares) and $128 \times 128$ pillars (diamonds). Strength thresholds are drawn according to Weibull distribution with index $\rho=2$. At least 1000 samples were taken for each presented value. The dashed lines represent function (6) with parameters computed from simulations 
Breakdown probability as a function of initial load per pillar $\sigma$ can be well approximated (dotted lines in Figs. 6 and 7) by the function:

$$
P(\sigma)=\frac{1}{2} \operatorname{erfc}\left(-\frac{\sigma-\xi}{\sqrt{2} \omega}\right)-2 \mathrm{~T}\left(\frac{\sigma-\xi}{\omega}, \alpha\right)
$$

where $\xi, \omega, \alpha$ are coefficients obtained from simulation results, $\operatorname{erfc}(z)$ represents complimentary error function

$$
\operatorname{erfc}(z)=1-\operatorname{erf}(z)=\frac{2}{\sqrt{\pi}} \int_{0}^{z} \exp \left(-t^{2}\right) d t
$$

and $\mathrm{T}(x, a)$ is Owen's $\mathrm{T}$ function which calculates

$$
\mathrm{T}(x, a)=(2 \pi)^{-1} \int_{0}^{a} \frac{\exp \left\{-\frac{1}{2} x^{2}\left(1+t^{2}\right)\right\}}{\left(1+t^{2}\right)} d t(-\infty<x, a<+\infty)
$$

Formula (6) is in fact a cumulative distribution function of the skew normal distribution with parameters: location $\xi$, scale $\omega$ and shape $\alpha$. So, there is an evident connection between the distribution of critical loads in a quasi-static procedure and the breakdown probability of the systems with the applied finite force.

In the last part we analyse results for systems with pillar-strength-thresholds truncated below $\sigma_{0}$ i.e. an initial load per pillar.

Figure 8 illustrates cascade and breakdown probabilities as a function of load $\sigma_{0}$. It is seen that cascade probability $P_{c}$ initially increases while breakdown probability $P_{b}$ is almost zero, but as the $\sigma_{0}$ grows, the $P_{b}$ rises more rapidly than $P_{c}$. For $\sigma_{0}>0.9$ the probabilities of breakdown and cascade start to converge - the formation of cascade is nearly tantamount to system breakdown.

Formula (6) with adequate coefficients can serve as an approximate function of breakdown probability and cascade probability in the case of truncated distributions (see Figs. 8 and 9). One exception to this is $P_{c}$ with truncated uniform distribution. We have found that $P_{c}$ for truncated Weibull distributions can also be nicely fitted by the formula

$$
P_{c}\left(\sigma_{0}\right)=1-\exp \left\{-\left(\varphi \sigma_{0}\right)^{\eta}\right\}
$$

where $\varphi, \eta$ are coefficients obtained from the simulation results. Graphically it has been shown in Figure 10. Results of cascade probability for truncated uniform 
distributions are also included, but none of the presented fitting functions can be used as an approximate formula.

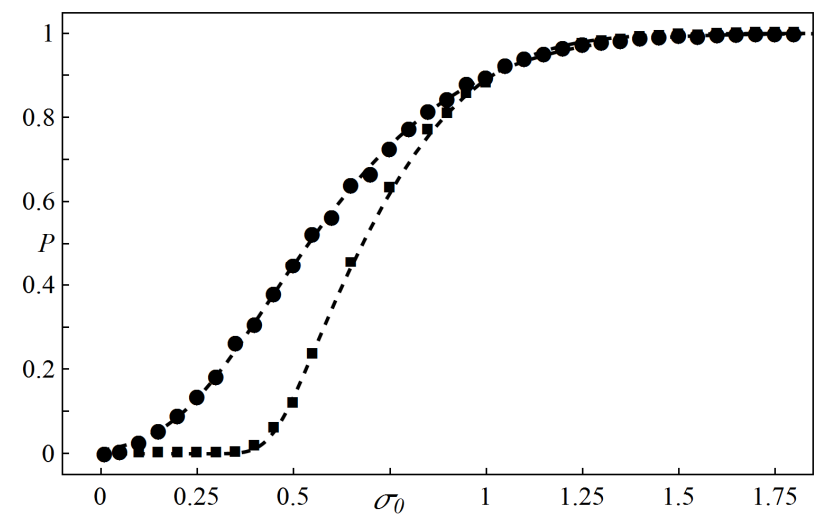

Fig. 8. Empirical: breakdown (squares) and cascade (circles) probabilities as a function of initial load per pillar $\sigma_{0}$ for system of $64 \times 64$ pillars. Strength thresholds are drawn according to Weibull distribution truncated below $\sigma_{0}(\rho=2)$. The results are taken from 2000 samples for each presented value. The dashed lines represent function (6) with parameters computed from simulations

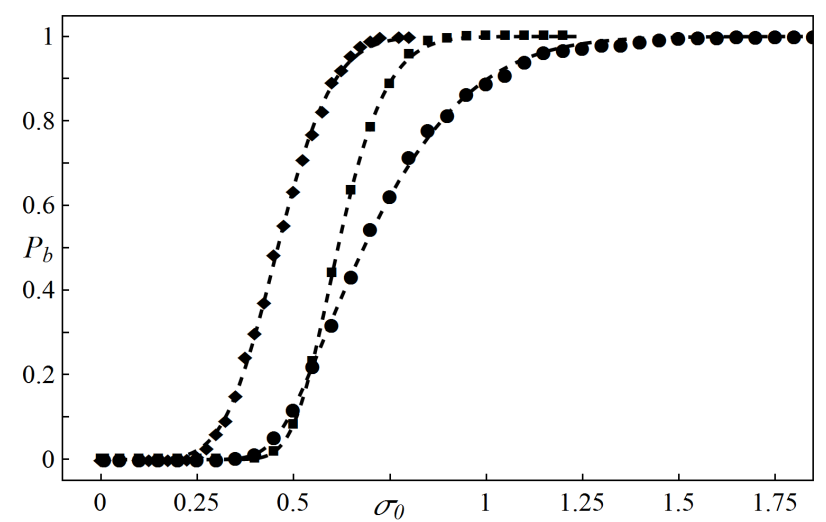

Fig. 9. Empirical breakdown probability $P_{b}$ as a function of initial load per pillar $\sigma_{0}$ for systems with truncated Weibull distributions: $\rho=2$ (circles), $\rho=5$ (squares) and truncated uniform distribution (diamonds). The results of the loading process of $N=128 \times 128$ pillars (square arrangement) are taken from 2000 realizations for each presented value. The dashed lines represent function (6) with parameters computed from simulations 


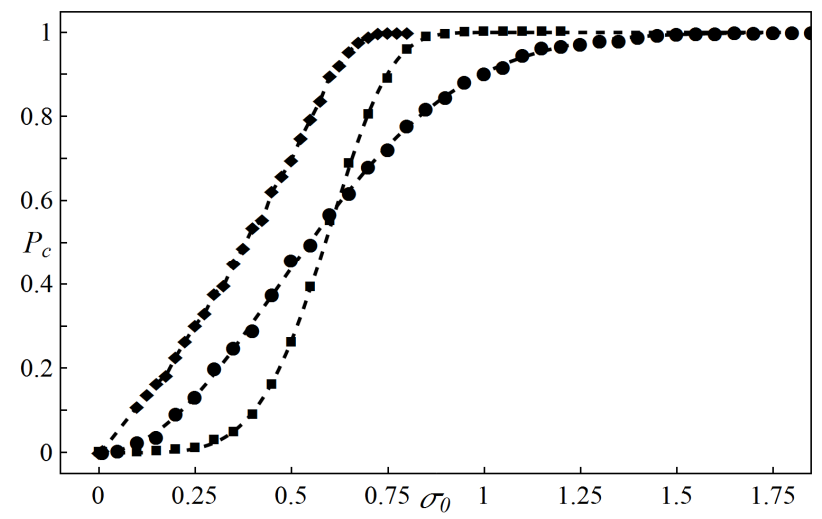

Fig. 10. Empirical cascade probability $P_{c}$ as a function of initial load per pillar $\sigma_{0}$ for systems with truncated Weibull distributions: $\rho=2$ (circles), $\rho=5$ (squares) and truncated uniform distribution (diamonds). The results of the loading process of $N=128 \times 128$ pillars (square arrangement) are taken from 2000 realizations for each presented value. The dashed lines for systems with truncated Weibull disorder represent function (9) with parameters computed from simulations

\section{Conclusions}

In this paper, we have introduced stochastic local load redistribution in the fibre bundle model of nanopillars arrays. Two different, but equivalent loading types are considered: quasi-static and finite force.

Based on the simulation results for quasi-static loading, we have noticed significant system strength decrease in comparison to the classical local load sharing. We have also reported that critical load and the number of nearest neighbours in a given lattice type are positively dependent. Values of critical load and catastrophic avalanche sizes follow a skew normal distribution.

For the procedure with the applied finite force, we have found that probabilities of breakdown and cascade are well fitted by the formula (6) which is a cumulative distribution function of the skew normal distribution. We also propose formula (9) as a fitting function for the cascade probability in case of pillar-strength-thresholds drawn according to a truncated Weibull distribution.

\section{References}

[1] Herrmann H.J., Roux S. (eds.), Statistical Models for the Fracture of Disordered Media, North Holland, Amsterdam 1990 and references therein.

[2] Chakrabarti B., Benguigui L.G., Statistical Physics of Fracture and Breakdown in Disordered Systems, Clarendon Press, Oxford 1997.

[3] Alava M.J., Nukala P.K.V.V., Zapperi S., Statistical models of fracture, Adv. in Physics 2006, 55, 349-476. 
[4] Pradhan S., Hansen A., Chakrabarti B.K., Failure processes in elastic fiber bundles, Rev. Mod. Phys. 2010, 82, 499-555.

[5] Biswas S., Ray P., Chakrabarti B.K., Statistical Physics of Fracture, Breakdown, and Earthquake: Effects of Disorder and Heterogeneity, Wiley, 2015.

[6] Hansen A., Hemmer P.C., Pradhan S., The Fiber Bundle Model: Modeling Failure in Materials, Wiley, 2015.

[7] Kun F., Raischel F., Hidalgo R.C., Herrmann H.J., Extensions of fibre bundle models, Lecture Notes in Physics 2006, 705, 57-92.

[8] Uchic M.D., Dimiduk D.M., Florando J.N., Nix W.D., Sample dimensions influence strength and crystal plasticity, Science 2004, 305 (5686), 986-989.

[9] Greer J.R., Jang D., Kim J.-Y., Burek M.J., Emergence of new mechanical functionality in materials via size reduction, Adv. Functional Materials 2009, 19, 2880-2886.

[10] Wang Z.-J., Li Q.-J., Shan Z.-W., Li J., Sun J., Ma E., Sample size effects on the large strain bursts in submicron aluminum pillars, Appl. Phys. Lett. 2012, 100, 071906.

[11] Derda T., Avalanche statistics in transfer load models of evolving damage, Scientific Research of the Institute of Mathematics and Computer Science 2011, 10(1), 21-31.

[12] Domański Z., Derda T., Sczygiol N., Critical avalanches in fiber bundle models of arrays of nanopillars, Proceedings of the International MultiConference of Engineers and Computer Scientists 2013, Vol II, IMECS 2013, March 13-15, 2013, Hong Kong.

[13] Domański Z., Derda T., Sczygiol N., Statistics of critical avalanches in vertical nanopillar arrays, Lecture Notes in Electrical Engineering 2014, 275, 1-11.

[14] Dalton F., Petri A., Pontuale G., A random neighbour model for yielding, Journal of Statistical Mechanics: Theory and Experiment 2010, P03011.

[15] Lehmann J., Bernasconi J., Breakdown of fiber bundles with stochastic load-redistribution, Chemical Physics 2010, 375 (2-3), 591-599. 